\title{
Community Detection Algorithm based on Centrality and Node Closeness in Scale-Free Networks
}

\author{
Sorn Jarukasemratana \\ Tokyo Institute of Technology \\ sorn.jarueai.cs.titech.ac.jp \\ Tsuyoshi Murata \\ (affiliation as previous author) \\ muratalcs.titech.ac.jp \\ Xin Liu ${ }^{* 1}$ \\ Tokyo Institute of Technology, CREST JST, Wuhan University of Technology \\ tsinlleweai.cs.titech.ac.jp
}

keywords: community detection, scale-free networks

\section{Summary}

We present a method for detecting community structures based on centrality value and node closeness. Many real world networks possess a scale-free property. This property makes community detection difficult especially on the widely used algorithms that are based on modularity optimization. However, in our algorithm, communities are formed from hub nodes. Thus communities with scale-free property can be identified correctly. The method does not contain any random element, nor requires pre-determined number of communities. Our experiments showed that our algorithm is better than algorithms based on modularity optimization in both real world and computer generated scale-free datasets.

\section{Introduction}

Community detection has become an interesting topic during the past years. Detecting community is useful because members in the same community usually share the same attributes [Fortunato 10]. For example, in proteinprotein interaction networks, each community of proteins has different functions for body. One of the important concept on community detection is modularity, offered by Newman and Girvan [Girvan 02]. Modularity is a measurement on how network is partitioned into communities. High modularity score means there are dense connections between the nodes within the same community, but sparse connections among different communities. Many community detection algorithms [Fortunato 10] aim to increase the modularity score by using modularity optimization methods. However, in some cases, high modularity score does not represent good communities. We found out that modularity optimization based algorithms are less accurate on scale-free networks which contain communities with a scale-free property.

Since the introduction of scale-free networks by Barabási

$\dagger 1$ Xin Liu gratefully acknowledges support from CREST JST and from NSFC under grant number 61203154.
[Barabási 03], a number of researchers claimed that many real world networks contain a scale-free property. For example, social networks, citations networks, the World Wide Web, the internet infrastructures, or biological networks are considered as scale-free networks. The main characteristic of scale-free networks is that the degree distributions of the network follows the power-law, and the degree distribution on a log-log scale can be seen as a straight line. This means there are many low degree nodes and only some high degree nodes. The high degree nodes are often called hubs. Hubs are normally connected with lower degree nodes which are then connected to even lower degree nodes and so on. We believe that this scale-free characteristic should be used when performing community detection on scale-free networks.

The problem of modularity optimization based algorithms on scale-free networks occurs when low degree nodes form a strongly connected group inside a community. Even if they are connected by hub nodes, if the number of edges in this strongly connected group is high enough, the algorithm will declare this sub-community as a new community. This behavior can break a community with a scalefree property into many sub-communities. This problem occurs because in most modularity optimization based al- 


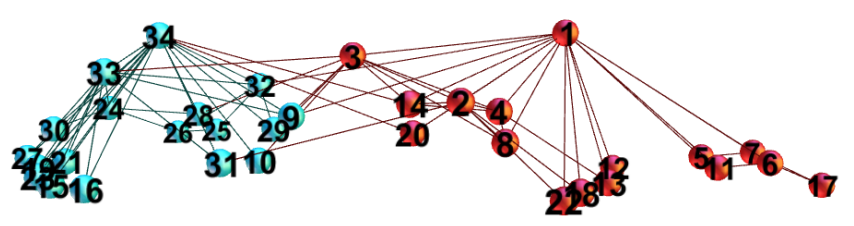

Fig. $13 \mathrm{D}$ visualization of Zachary's karate club. X-Y plane layout is generated by force atlas, $\mathrm{Z}$ axis is centrality value of each node.

gorithms, all nodes and edges are treated equally. However, in our opinion, that is not the accurate way. In scalefree networks, nodes with high degree or hubs should be considered more important than these with lower degree. Moreover, edges that originated from hubs should be considered more important than those that connect the lower degree nodes.

In this paper, we present a community detection algorithm which uses centrality and node closeness to determine communities. There are two main ideas for this algorithm. The first idea is that community should be formed from the nodes that play important roles in a network. The second idea is that two nodes should be in the same community if they are closely connected together. By using centrality value, the hub nodes that are considered important in scale-free networks can be identified correctly. Then, community detection is started from the highest centrality node to the lowest centrality node. Node closeness is used to determine if two nodes should be in the same community or not.

We compare our method with the Louvain algorithm, one of the most popular community detection algorithms (this algorithm is also known as fast unfolding). Our algorithm can achieve higher NMI value than Louvain algorithm in scale-free networks. Moreover, when our algorithm is displayed with force-directed layout as $x-y$ plane and our algorithm's centrality values are used as $\mathrm{z}$ axis, the scale-free community shows a mountain shape structure. Figure 1 is a 3D visualization of our algorithm on Zachary's karate club network [Zachary 77]. Two communities can be observed by two mountain shape structures. The nodes at the peak of the mountain are the hub nodes.

This community detection algorithm is similar to pouring colored water onto the peak of each mountain, where each color represents a community. For example, we pour red water onto the top of the highest mountain peak (node 1 in figure 1). The water will flow to its closest node (node 2 ). The second highest node is node 34 . Since it is the peak of the mountain, we pour new color on it, in this case blue. As our algorithm continues, the water flows down from the top of the mountain until it reaches the base. Fi- nally, the whole mountains are covered with color and our algorithm is finished. Similar to a characteristic of water that it will not flow up, our algorithm is following this characteristic as well. This means the node that has higher centrality value will not be assigned community based on lower centrality nodes.

This paper is organized as follows. In section 2, related works are reviewed. In section 3, our algorithm is explained. In section 4 , we showed our algorithm results compared with modularity optimization based algorithm. In section 5 , we provide a discussion from the results in section 4. The last section is the conclusion.

\section{RELATED WORK}

Many community detection algorithms have been introduced in order to detect and identify communities in graphs. This problem has been extensively studied for many years, by researches from many fields including graph theory, computer sciences, biology or physics. One of the most important stepping stones in community detection field is the introduction of the modularity score by Girvan and Newman [Girvan 02]. Modularity score is used to measure how well a network is partitioned into modular communities. High modularity score means there are dense connections between the nodes within the same community, but sparse connections among different communities. Since then, modularity score has quickly became the benchmark of community detection algorithms. Therefore, many algorithms are making every effort to increase the modularity score. Hence, the term "modularityoptimizing community detection algorithms" was invented to categorize these algorithms. One of the most popular algorithm in this genre is Louvain algorithm by Blondel et al. [Blondel 08]. The modularity score of this method has been tested and it is shown to be excellent in comparison with other methods. Louvain algorithm starts by randomly choosing a node in a graph and try to group it with another node. After finding the best modularity gain pair, these two nodes are grouped together. This process is repeated until total modularity is at the maximum. Then, the algorithm will start randomly choosing groups instead of nodes to find the maximum modularity gain. The algorithm is finished when the modularity is at the maximum and no groups can be merged to gain more modularity score.

Another index for measure the goodness of community detection is normalized mutual information or NMI. The NMI is used to compare two sets of partitioning results. The value is high when two results are similar. NMI is 
normally used when the ground-truth of the network (the correctly partitioned set) is available. This severely limits the use of NMI since many networks do not have groundtruth, such as friends networks or communication networks. However, NMI can be used on synthetic networks or some well-known networks that ground-truth communities are available.

There are also some community detection algorithms that are designed specifically for scale-free networks. Probably the first one of this kind is the work of $\mathrm{Wu}$ et al. [Borgatti 05], titled "Mining Scale-free Networks using Geodesic Clustering”. Geodesic distance or shortest path is used in their method called geodesic clustering. Mean geodesic distance is calculated, and it is used for selecting a node to be in the same group. Abou-Rjeili and Karypis [Abou-Rjeili 06] offered a multilevel algorithms for partitioning power-law graphs. The key idea of their algorithm is, instead of partitioning directly from the original graph, the approximations of original graph should be obtained first. The approximation can be done multiple times until the approximated graph is small enough to compute with any algorithm. Once partitioning is done, the last step is to derive partitioning results to the original graph. Qian et al. [Qian 07] used hypergraph for clustering scale-free networks. In a normal graph, an edge can only connect two nodes together. However, in a hypergraph, an edge can connect to any number of nodes, this edge is called hyperedge. In their work, they created hyperedges from the density of a graph to find the communities. Xu et al. [Xu 07] introduced SCAN: A Structural Clustering Algorithm for Networks. Their algorithm is working by identifying each node into many classes, such as core, hub, and outlier. Then, partitioning is done according to the roles of each node class.

\section{Our Algorithm}

There are two main ideas in our algorithm. The first idea is that nodes that are close together should be in the same community. The second idea is that the community should be formed from the important nodes. To determine the closeness among nodes, we use node closeness as the measurement. And to determine the importances of the nodes, we use node centrality. Once node closeness and node centrality are calculated, the community detection phase is employed. In this phase, all nodes are first treated as no community. Then, starting from the highest centrality node, the community is assigned to it and its closest neighbor. After that, the next highest centrality node and its closest neighbor are picked to be assigned the com- munity. The algorithm is finished when all nodes have been assigned to any of the community. This section is divided into four subsection: centrality value, node closeness, community detection, and configurable variables of the algorithm.

\subsection{Centrality Value}

Node centrality is one of the most intuitive method to identify the important nodes in a network. The early researches about node centrality can be dated since 1950s by Bavelas and Leavitt [Estrada 11]. In general, node centrality represents its ability to communicate with other nodes, or its closeness to other nodes. The most basic form of centrality is degree centrality. The main idea of this centrality is that the node is more central than others if it connects to more nodes. In short, degree centrality value is equal to node degree. The main flaw of degree centrality is that it only accounts the neighbor nodes. To correctly represent the centrality of nodes, the nodes that farther away should also be included into the calculation. Leo Katz [Katz 53] introduced his version of centrality measurement which is later known as Katz centrality. To calculate the degree centrality of node $i$, we count the nodes that are one hop away from node $i$ (thus this value is equal to the degree of node $i$ ). However, in Katz centrality, nodes that are $n$ hops away from node $i$ are also counted. The nodes that are $n$ hops away can be found by the $n$-th power of the adjacency matrix. However, nodes that take less hops to reach should provide more centrality score than the nodes that are farther away. So, the attenuation factor $\alpha$ is introduced. Katz centrality can be expressed as the equation below.

$$
K_{i}=\left[\left(\sum_{k=0}^{\infty} \alpha^{k} A^{k}\right) I\right]_{i}
$$

where $K_{i}$ is the Katz centrality of node $i, A^{k}$ represents the power $k$-th of the adjacency matrix, $\alpha$ is the attenuation factor and $I$ is the column vector where all values are 1 .

Consider normal scale-free networks; Katz centrality can identify hub nodes correctly since hubs normally have high degree. Even in the case where hubs do not have the highest degree itself, nodes from the second and third length hops are usually enough to increase the centrality score of hub nodes to a noticeable level. However, the problem is the node that connects at least two hubs together. This node will have a high centrality score from connecting to hubs and sometimes it may seem more important than the hub nodes. The example of this problem is shown in figure 2. To solve this problem, we have de- 


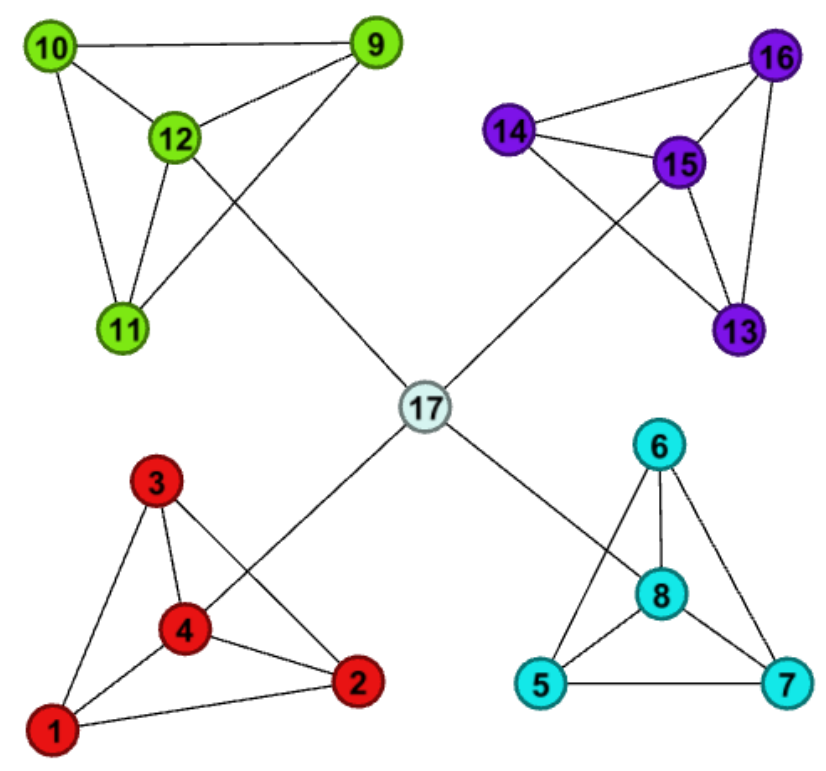

Fig. 2 Node 17 has the highest Katz centrality score. Without using subgraph centrality, the whole graph will merge into one community, while modularity-based algorithms will give 4 communities.

cided to add another centrality value to compliment the Katz centrality.

Subgraph centrality was introduced in 2005 by Estrada and Velázquez [Estrada 05]. Similar to Katz centrality, subgraph centrality also uses the hops length increment method, starting from 0 hop to $n$ hops. The main difference is that only the hops that complete the closed walk are counted. Closed walk of 0 hop (0 length) is the node itself. Closed walks of 1 hop (length 1) are not available (since it cannot hop back to the starting node to create a closed walk). Number of closed walks of length 2 are equal to its degree, the node hops to its neighbor nodes and hops back to itself to create closed walks. Closed walk of length three is a triangle shape closed walk. The length three closed walk from node $i$ involving node $j$ and $k$ would form a triangle shape like this; node $i \rightarrow$ node $j \rightarrow$ node $k \rightarrow$ node $i$. Subgraph centrality score of length $l$ on node $i$ can be calculated by this formula.

$$
f_{i}(A)=\left(\sum_{l=0}^{\infty} c_{l} A^{l}\right)_{i i}
$$

where $f_{i}$ is subgraph centrality of node $i, l$ is the length of closed walks, $c_{l}$ is the coefficient of length $l$ (similar to attenuation factor in Katz centrality), and $A^{l}$ represents the $l$-th power of the adjacency matrix. After applying subgraph centrality into graph in figure 2, node 12, 4, 8, and 15 become more important than node 17 . The number of communities now becomes 4 communities.
In scale-free networks, length three subgraph centrality score can clearly differentiate hub nodes from non hub nodes. This can be seen in figure $3 a$ where node 1 , a hub node, has 18 length three closed walks, the highest number in this network. This can solve the problem of Katz centrality on nodes that connect at least two hubs together. This is because those nodes do not participate in many length three closed walks. Since these two algorithms contain many overlapping parts, we choose to combine only the length three subgraph centrality with Katz centrality to create our centrality score. The formula of the centrality score is shown below;

$$
K_{i}=\left[\left(\sum_{k=0}^{\infty} \alpha^{k} A^{k}\right) I\right]_{i}+\left(c A^{3}\right)_{i i}
$$

Another point for discussion is how many hops should be used. In both Katz centrality and subgraph centrality, the number of hops is set to infinite. Katz centrality's attenuation factor and subgraph centrality's coefficient are selected so that the infinity converge. This is reasonable because centrality value should be calculated from the involvement of every node in a network. However, in our case, we only want the centrality value to identify the hubs. Therefore, it is not necessary to involve the nodes that are too far away. We observed from our experiment that length of three hops is the most appropriated value because it provides the best compromise between accuracy and computation time. However, it is possible to use higher hop number to increase accuracy at the cost of the computation time.

\subsection{Node closeness}

Node closeness is calculated to determine how close the two nodes are. Node closeness can be seen as an inverse of the node distance. Node closeness can be calculated from node distance matrix. Let $A$ be the distance matrix, the shortest distance or shortest path from node $i$ to node $j$ is equal to $A_{i j}$. However, communication between nodes does not always use the shortest path. For example, if we want to send a large amount of data from node $i$ to node $j$, we would use all the paths that are available to maximize the data flow. Node closeness is the value that used to measure this maximum data flow. Node closeness can also be regarded as a measure of how fast it will take to spread information from node $i$ to node $j$. Our node closeness is developed based on the Katz centrality algorithm. Node closeness matrix can be calculated by using the formula shown below;

$$
D_{i j}=c_{1} d_{i j 1}+c_{2} d_{i j 2}+\ldots+c_{l} d_{i j l}
$$


$D_{i j}$ is the node closeness between node $i$ and node $j . d_{i j l}$ is the total number of paths that has length of $l$ that connect node $i$ and node $j . c_{l}$ is the coefficient value. $l$ is the number of hops or lengths. The higher the node closeness score between node $i$ and node $j$ is, the closer they are.

Similar to Katz centrality, this value can identify the closeness between nodes very well, but it creates a problem in scale-free networks. The problem occurs on the nodes that are not the part of the community but are connecting at least two hubs together. The closeness score on those nodes can be very high, resulting in a merging of the two scale-free communities. The solution to this problem is similar to centrality case, which is to add the length three closed walk of subgraph centrality. Let node $i$ connects to node $j$ and node $k$. If node $j$ and node $k$ are connected, the length three closed walk is formed. Then, the closeness score from node $i$ to node $j$ and node $i$ to node $k$ will be increased.

Probably the most beneficial factor about using this node closeness is that it is a byproduct of the centrality value. In fact, the sum of the closeness value from node $i$ to other nodes in graph is equal to our centrality value, $K_{i}$ $=\sum_{j=0}^{\infty} D_{i j}$. Therefore, the calculation needs to be done only once to get both centrality score and node closeness score. The $l$ value of node closeness is also similar to the $l$ value used in centrality calculation.

\section{$3 \cdot 3$ Community detection}

At this stage, we already obtained centrality value of each node and the node closeness matrix of the network. To start the community detection phase, we treat all nodes as no community. Then we pick the node that has the highest centrality value as our main node and its closest node as the secondary node. The closest node refers to the node that has the highest closeness score from the main node. This node can be easily looked up from our closeness matrix. Then we have to check if our main node or secondary node already have a community or not. There are 4 possibilities; 1) if the main node already has a community but the secondary node does not, the secondary node will join the same community as the main node. 2) if the main node does not have community but the secondary node already has the community, the centrality score will be used to determine the outcome of this situation. If the main node's centrality score is higher than the secondary node's, a new community will be created and assigned to the main mode. But if the main node's centrality score is lower than the secondary node's, the main node will be assigned to the same community as the secondary node. 3 ) if both nodes do not have any community assigned, a new community will be created and assigned to both nodes. 4) if both nodes already has community, nothing will be done. This process starts from the highest centrality node to the lowest centrality node. A constraint relaxing parameter called laxing value is also used in this algorithm. This parameter is explained in the next section. The pseudo code of this algorithm is shown in algorithm 1.

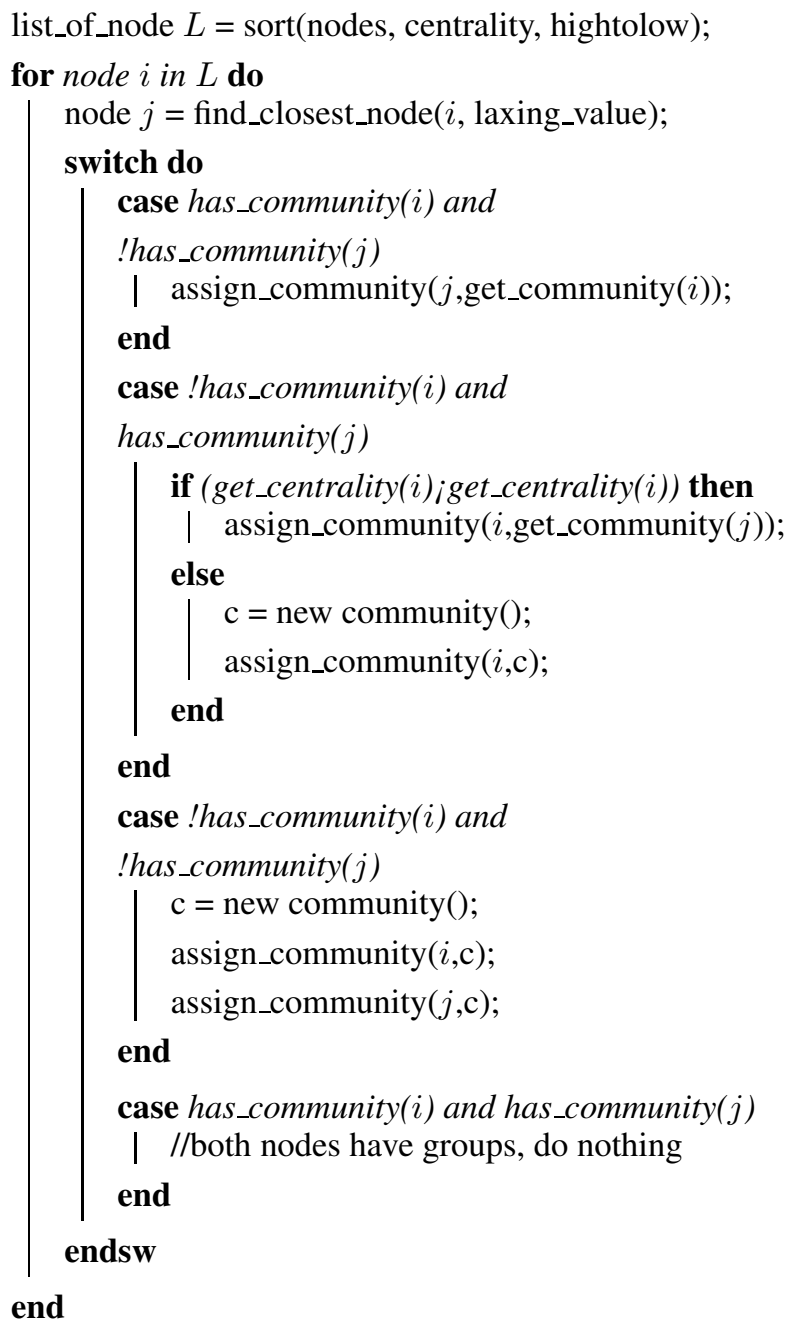

Algorithm 1: Community detection algorithm

The characteristic of this algorithm is similar to a flow of water. When we visualize a network in 3D by using force layout algorithm (in this case, we used force atlas 2 [Jacomy 11]) and assign the $\mathrm{z}$ axis value as our centrality value, the scale-free community become a mountain shape structure with the hub node as its peak. Figure 1 is the 3D representation of our algorithm on the Zachary's karate club network. It can be seen that there are two scale-free communities in this network (two mountains). The peaks of those mountains are Mr. $\mathrm{Hi}$ and John, the leader of each group. The communities are spreading from these 2 nodes. The result is that we have 2 communities that have $\mathrm{Mr}$. Hi and John as the center of each community. Also, 


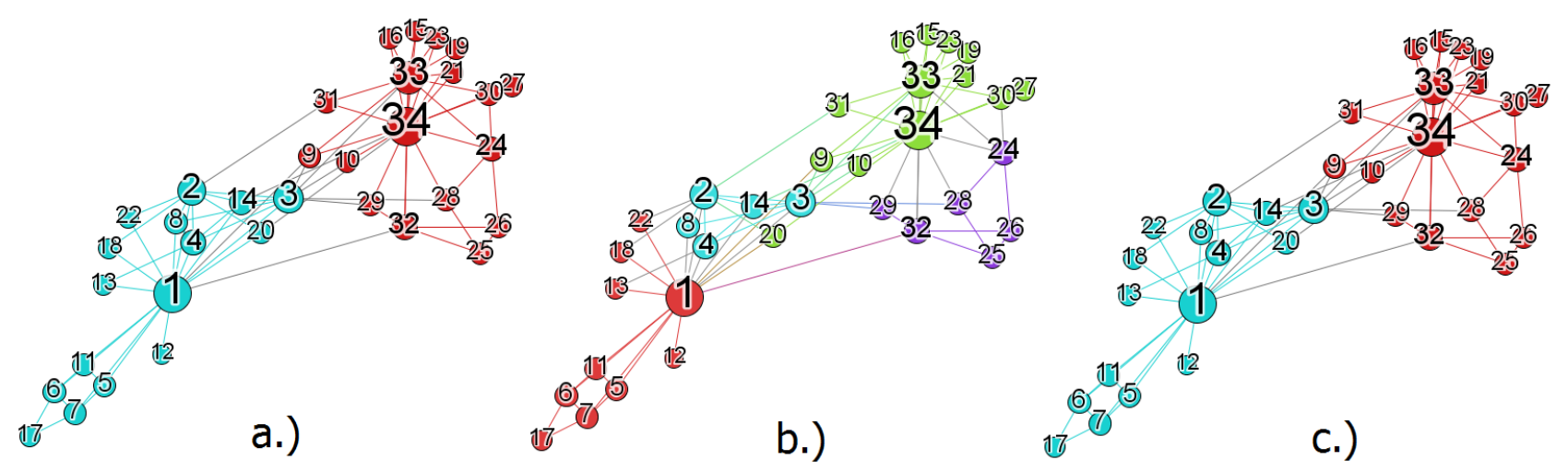

Fig. 3 Figure of Zachary's karate club a.) truth value b.) Louvain algorithm - NMI $=0.3375$, c.) our algorithm - NMI $=$ 1.0

similar to water that it will not flow up, the condition 2 in our pseudo code is representing this characteristic.

\section{$3 \cdot 4$ Configurable parameters}

There are several configurable parameters in this algorithm, which are the coefficient value or the attenuation factor $c$, the depth of the algorithm or the maximum number of hops $l$, and the constraint relaxing parameter called laxing value. Each will be explained about the effect of these values.

In Katz centrality, coefficient or attenuation value must not be set to the eigenvalue of the network. This is because it is required that the matrix to converge when number of hops is set to infinite. However, since we choose not to use the infinite number of hops, this restriction does not apply. Coefficient value is the value that act as a score reduction on nodes that are farther away. This value is the same on both closeness and centrality. For example, suppose coefficient value is set at 0.1 . Let node $i$ connects to node $j$, and node $i$ has a degree of 1 . Node $j$ connects to 3 nodes or has a degree of 3 . The final score of node $i$ is $1 *$ $0.1^{0}+3 * 0.1^{1}=1.3$ point. Different coefficient value can change the outcome of the final score. For example, comparing two nodes, let node $i$ connects to 2 nodes that are one hop away and 2 nodes that are two hops away, while node $j$ connects to 1 nodes that are one hop away and 7 nodes that are two hops away. If coefficient is 0.1 , node $i$ score is $2.2\left(2 * 0.1^{0}+2 * 0.1^{1}\right)$ while node $j$ score is 1.7 $\left(1 * 0.1^{0}+7 * 0.1^{1}\right)$. However, if coefficient is 0.4 , the scores will become $2.8\left(2 * 0.4^{0}+2 * 0.4^{1}\right)$ and $3.8(1 *$ $\left.0.4^{0}+7 * 0.4^{1}\right)$. This can drastically change the outcome of the algorithm. In scale-free networks, the degree distribution of the networks follows a power-law. The coefficient value should be set low such as 0.1 . This is because in scale-free networks hub nodes usually have high degree and many length three close walks, which is enough to distinguish hub nodes from normal nodes. However, in networks that degree distribution follows the poisson distribution such as random networks or small-world networks, the coefficient should be set to high value such as 0.4. This makes centrality value on important nodes become more distinguishable from other nodes. Combining this change with another configurable parameters, laxing value, our community detection algorithm can work correctly on non scale-free networks as well.

The number of hops or $l$ also plays an important role in this algorithm. It is noted that $l$ value must be more than three in order to use the length three closed walk as a part of the calculation. In the case of $l$ is equal to one, centrality will become degree centrality and node closeness will be only in 0 (not connected) and 1 (connected). The number of hops greatly affects the complexity of the algorithm. This will be explained in section 5 .

The last configurable parameter is laxing value. Laxing value is used in community detection phase. The role of laxing value is to make a group of nodes that have similar closeness scores to act as one node. For example, node $i$ is the main node and node $j$ is the secondary node with closeness score of 9.9 and node $i$ already has a community. According to our algorithm, node $j$ should join node $i$ 's community. However, if there is node $k$ that is also connected to node $i$ but has a closeness value of 9.8, since node $k$ is very similar to node $j$ (9.9 and 9.8 score), node $k$ should also be given a community from node $i$ similar to node $j$. In this case the laxing value will group node $k$ and $j$ together, then node $i$ will assign community to both of them. Laxing value is a percentage based value. For example, laxing value is set at $95 \%$. Let node $j$ has closeness score of 9.9 , node $k$ has 9.8 , and node $m$ has 9.3. Node $j$ and $k$ will be grouped together because node $k$ is more than $95 \%$ of the highest score (in this case more than $95 \%$ of 9.9) while node $m$ will be neglected. In a network that has scale-free property, this value is set as $95 \%$ in this research. However, in a network that has no clear 
Table 1 NMI of Louvain algorithm and our algorithm on various networks. (the higher the better)

\begin{tabular}{|c|l|l|}
\hline \multicolumn{1}{|c|}{ Networks } & Louvain & Ours \\
\hline Zachary's karate club & 0.3375 & 1.0 \\
\hline Dolphins network & 0.3258 & 0.6028 \\
\hline Synthetic network 1 & 0.3538 & 0.5208 \\
\hline Synthetic network 2 & 0.48 & 0.289 \\
\hline Football network & 0.7535 & 0.7897 \\
\hline Political books network & 0.3461 & 0.507 \\
\hline
\end{tabular}

central node like small-world networks, this value is very important for grouping nodes together into communities. We found out that $90 \%$ is enough to provide a solid result on non scale-free networks. Noted that if this value is set to $0 \%$, the community will spread from the main node through all edges to all of its connected nodes, without considering the closeness value.

Another configurable aspect that should be mentioned is that the method for calculating centrality value and node distance value can be changed. There are many proven centrality calculation algorithms such as eigenvector centrality [Bonacich 07], closeness centrality [Borgatti 05], betweenness centrality [Freeman 77] and more. All of these centrality values can be exchanged with our centrality value to create another community detection result. Similar to centrality value, our closeness value algorithm can be replaced with other algorithms as well, such as the inverse value of resistance distance [Klein 93].

\section{Experiment}

Comparing the results of the partitioning with groundtruth partition by using NMI is probably the most accurate method to grade the result of the partitioning. Many NMI algorithms are available, but in this research, we use generalized normalized mutual information developed by Lancichinetti et al. [Lancichinetti 09]. One disadvantage of this method is that the ground-truth information of the network is required. This is especially hard for many real world networks since there is no correct way to categorize the dataset. However, there are some famous real world networks where ground-truth value is available and often used in testing the validity of the community detection algorithms [Fortunato 10]. Networks that are used in this paper are Zachary's karate club [Zachary 77], Doubtful Sound dolphin network [Lusseau 03], American college football network [Girvan 02], and political books network [Krebs 04]. In addition to the known famous networks, synthetic networks are another options. In order to generate scale-free networks, we use the method describe in "Benchmark Graphs for Testing Community Detection Algorithms" by Fortunato [Lancichinetti 08]. Since we aim only to partition the unweighed undirected graph, the basic version of Fortunato's work is used. To test with more real world datasets, we used ego-Facebook datasets provided by Stanford Large Network Dataset Collection [McAuley 12], however, the ground-truth value for egoFacebook datasets is not available. To validate the accuracy of our method, we compared our results with modularity optimization based algorithm called Louvain algorithm or as known as fast unfolding. It is shown in their research that it surpassed other modularity optimization methods.

The truth value of Zachary's karate club network is extracted from the node attributes table presented in "An Information Flow Model for Conflict and Fission in Small Groups" by Wayne W. Zachary [Zachary 77]. There are five categories of factions: Mr. Hi - Strong, Mr. Hi Weak, John - Strong, John - Weak, and None. We then assign John - Strong and John - Weak to community 1 and Mr. Hi - Strong and Mr. Hi - Weak to community 2. Nodes without faction are neglected. The degree distribution of this network is shown as almost straight line in log-log scale. Power-law degree distribution can be observed. As shown in figure 3, a network got separated into 5 communities by Louvain method while our algorithm provided 2 communities similar to the truth value. In result, the NMI value of our algorithm is significantly higher. Also by looking at figure 1 , it is shown that our algorithm can detect 2 mountain shape structures. Each mountain represents a community with scale-free property.

The Bottlenose dolphins of Doubtful Sound network is another frequently used by community detection researchers. Unlike Zachary's karate club, the known truth value is not available. However, in consensus, this dolphins network is believed to be divided into 2 communities as shown in figure 4a. With Louvain algorithm, a network was split into 5 communities while our algorithm provided 3 communities, and the truth value contains 2 communities. Our algorithm also produced higher score in NMI. In 3D visualization, our algorithm detected 3 mountain shape structures, suggesting 3 scale-free communities.

We used Fortunato's work to create benchmark graphs [Lancichinetti 08]. The graph follows the power-law on both node's degree distribution and community size. The program can be downloaded directly from Fortunato's website [Fortunato 13]. Two synthetic networks are shown. The sizes of the networks are 200 and 300 nodes respectively. The result can be seen in figure 5. In network 1, our algorithm can identify the number of communities cor- 

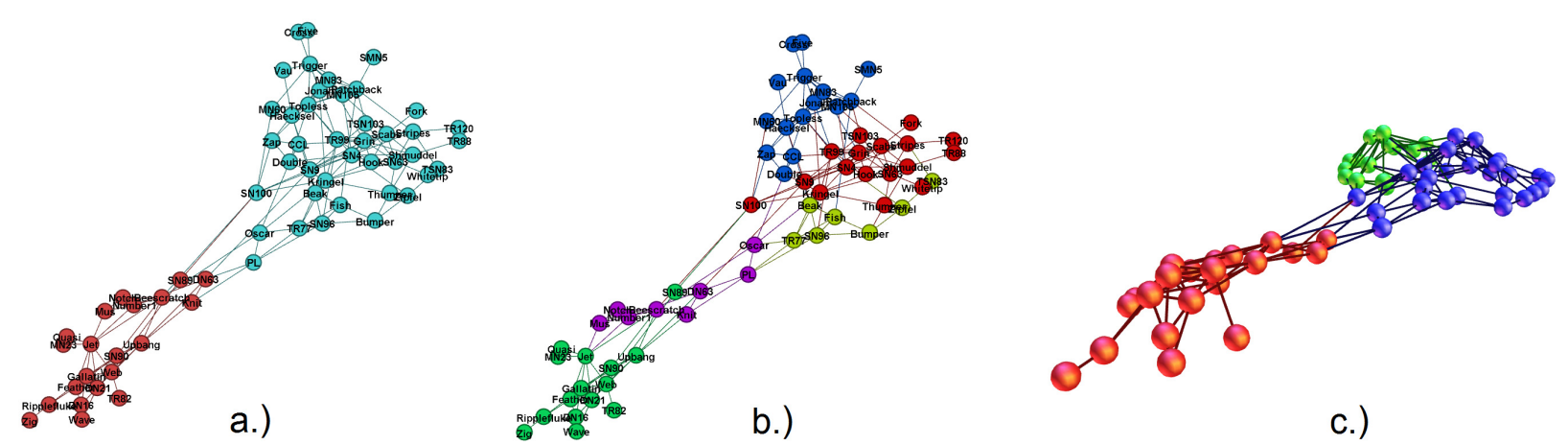

Fig. 4 Figure of dolphins, a.) truth value b.)Louvain $-\mathrm{NMI}=0.3258$, c.) our algorithm (in 3D visualization) $-\mathrm{NMI}=$ 0.6028

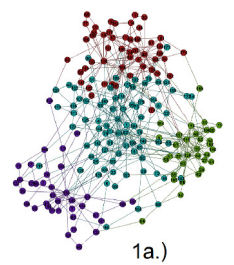

1a.)

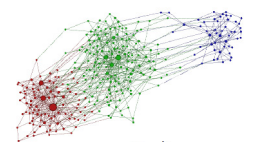

2a.)

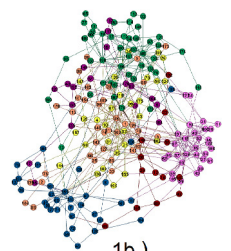

1b.)

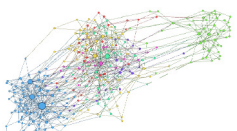

2b.)

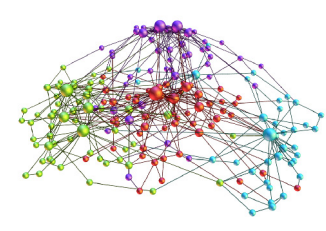

1c.)

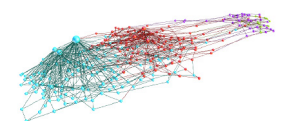

2c.)

Fig. 5 Figure of network 1 and network 2, 1a.) truth value 1b.)Louvain algorithm $-\mathrm{NMI}=0.3538,1 \mathrm{c}$.) our algorithm $-\mathrm{NMI}=0.5208$ , 2a.) truth value $2 \mathrm{~b}$.)Louvain algorithm $-\mathrm{NMI}=0.48,2 \mathrm{c}$.) our algorithm $-\mathrm{NMI}=0.289$

rectly which is 4 , and provided higher NMI value. However, in network 2 (figure 5-2b), our algorithm could identify the left and middle communities correctly but did not perform well on the right community. The reason for this is because the right community does not possess a scalefree attribute. Therefore, our algorithm failed to identify it as one community. This can be seen in the $3 \mathrm{D}$ visualization (figure 5-2c) as a flat area on the far right. This problem will be discussed further in the section 5 .

Scale-free networks are only a small portion of total networks. In networks that has poisson distribution such as random networks or small world networks, modularity optimization is probably the best method to detect communities on those networks. Modularity score also has a great advantage over NMI, which is modularity score does not require a ground-truth value. Since, our algorithm is aimed for scale-free community detection, it does not perform well on non scale-free networks. However, in order for our algorithm to be used on non scale-free networks, we have to modify our configurable parameters so that our algorithm can perform a community detection effectively. Two non scale-free datasets are used in this section, American college football network [Girvan 02], and US political

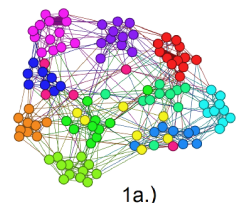

1a.)

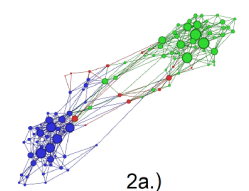

2a.)

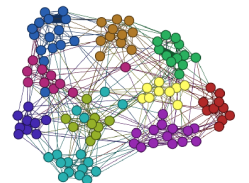

1b.)

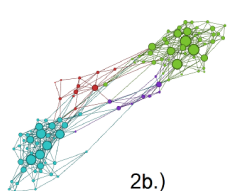

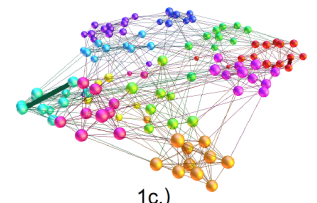

1c.)

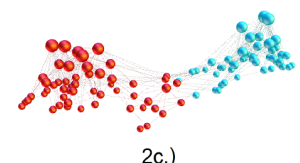

Fig. 6 Figure of American college football network (1) and US political books network (2), 1a.) truth value 1b.)Louvain - NMI = $0.7535,1 \mathrm{c}$.) our algorithm - NMI $=0.7897,2 \mathrm{a}$.) truth value 2b.) Louvain $-\mathrm{NMI}=0.3461,2 \mathrm{c}$.) our algorithm $-\mathrm{NMI}=0.507$

books network [Krebs 04]. The $c$ value is set to 0.4 and laxing value is set to $90 \%$ in our algorithm.

As mentioned in the algorithm section, setting coefficient value to 0.4 will greatly increase the score of the nodes in the same community. This is because nodes in the same community are tightly connected to each others. Moreover, laxing value is also reduced to the level that nodes with similar closeness score are considered to be the same node. As in figure 6-1c and 6-2c, nodes from tightly connected group became the hubs of the community.

To show the result of our algorithm on real scale-free social networks, we used a portion of ego-Facebook datasets provided by Stanford Large Network Dataset Collection [McAuley 12], namely, network number 0, 384, 1648, and 3473. The result can be seen in figure 7 . Network 0 and network 384 showed one huge scale-free community and a few smaller communities. Network 1648 showed two large scale-free communities with a few smaller communities. Network 3437 showed one large scale-free communities and several medium/small size communities. It should be noted that there are contradictions between our algorithm and Louvain algorithm in every graphs, especially on the largest community. Louvain algorithm tended to separate the largest community into several smaller com- 
munities, while our algorithm detects only one scale-free community. In 3D visualization, the mountain shape structures can be seen in all of the Facebook graphs.

\section{Discussion}

It can be observed from our experiment that modularity optimization based algorithms can sometimes break a community with scale-free attribute into many communities; for example, both communities on Zachary's karate club (figure $3 b$ ), both communities on dolphins network (figure 4b), and communities on synthetic networks (figure 5-1b and 5-2b). Considering the definition of modularity, the breakdown of these scale-free communities can obtain higher modularity score. This phenomenon occurs when low degree nodes form a tightly connected group within a scale-free community, although not every scalefree networks have this phenomenon. The reason behind this occurrence is that modularity based algorithms considered all edges and nodes as equal. This characteristic makes modularity optimization based methods less suitable for scale-free networks. In our opinion, hub nodes and edges that generated from hub nodes should be treated with more importance than other nodes and edges. Our algorithm is implemented based on this idea and the result is presented in this work.

The flaw of our algorithm is shown in the synthetic network 2 (figure 5-2c), especially on the far right community, which does not contain a scale-free property. We have shown that our algorithm can work on non scalefree network in previous section by modifying our configurable parameters. However, what will happen when there are scale-free communities and non scale-free communities in the same network? With our current implementation, we can first identify scale-free communities with the parameters setup for scale-free. Then we run our algorithm again with the setup for non scale-free. This solution is still not satisfactory and still requires further research.

Another discussion that should be mentioned is the complexity of the algorithm. Originally, our algorithm's complexity is at $\mathrm{O}\left(\mathrm{n}^{3}\right)$. This is the complexity of the most time consuming part in our algorithm, the Katz centrality. However, in 2001, Foster and Muth [Foster 01] introduced "A Faster Katz Status Score Algorithm" which can reduced the complexity to $\mathrm{O}(\mathrm{n}+\mathrm{m})$, by using list instead of matrix. After applying the idea from Foster and Muth research, the complexity of our algorithm became $\mathrm{O}\left(\mathrm{mk}^{l-1}\right)$ where $\mathrm{m}$ is the number of edges, $\mathrm{k}$ is the average degree, and $l$ is the number of hops. Since we use $l$ equals to 3 , the effective complexity is $\mathrm{O}\left(\mathrm{mk}^{2}\right)$. The cur-

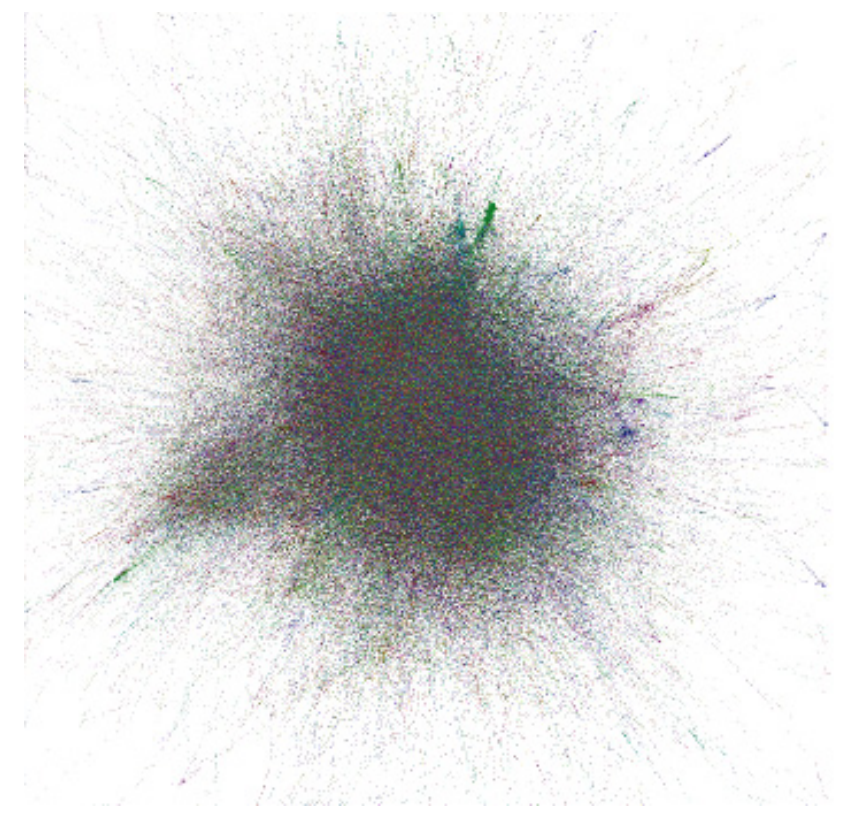

Fig. 8 Result of our algorithm on large scale network with more than 300,000 nodes and 1,000,000 edges; total computation time is 26 minutes.

rent largest dataset which was tested with this algorithm contains 317,080 nodes and 1,049,866 edges. The total computation time is 26 minutes on a laptop computer using Intel i7 with $8 \mathrm{~GB}$ of RAM. The result is shown in figure 8 .

\section{Conclusions}

We have introduced a community detection algorithm based on centrality and node closeness value. The algorithm works well on scale-free networks, and can score higher NMI scores when compared with modularity optimization based algorithms. The first main idea of this algorithm is that the community should be formed with the nodes that play important roles in a network; in this case, the hub nodes. The centrality value is used to find the hubs of each community and to use it as a community starting point. Node closeness value is used to determine the closest connected node. These are then applied to the second main idea of our algorithm; nodes which are closely connected together should be in the same community. Moreover, if we use the centrality value calculated in our algorithm as the $\mathrm{z}$ axis and then visualize the graph in $3 \mathrm{D}$, the community with scale-free property can be seen as a mountain shape structure.

\section{$\diamond$ References $\diamond$}

[Abou-Rjeili 06] A. Abou-Rjeili and G. Karypis: Multilevel algorithms for partitioning power-law graphs. In Proceedings of the 


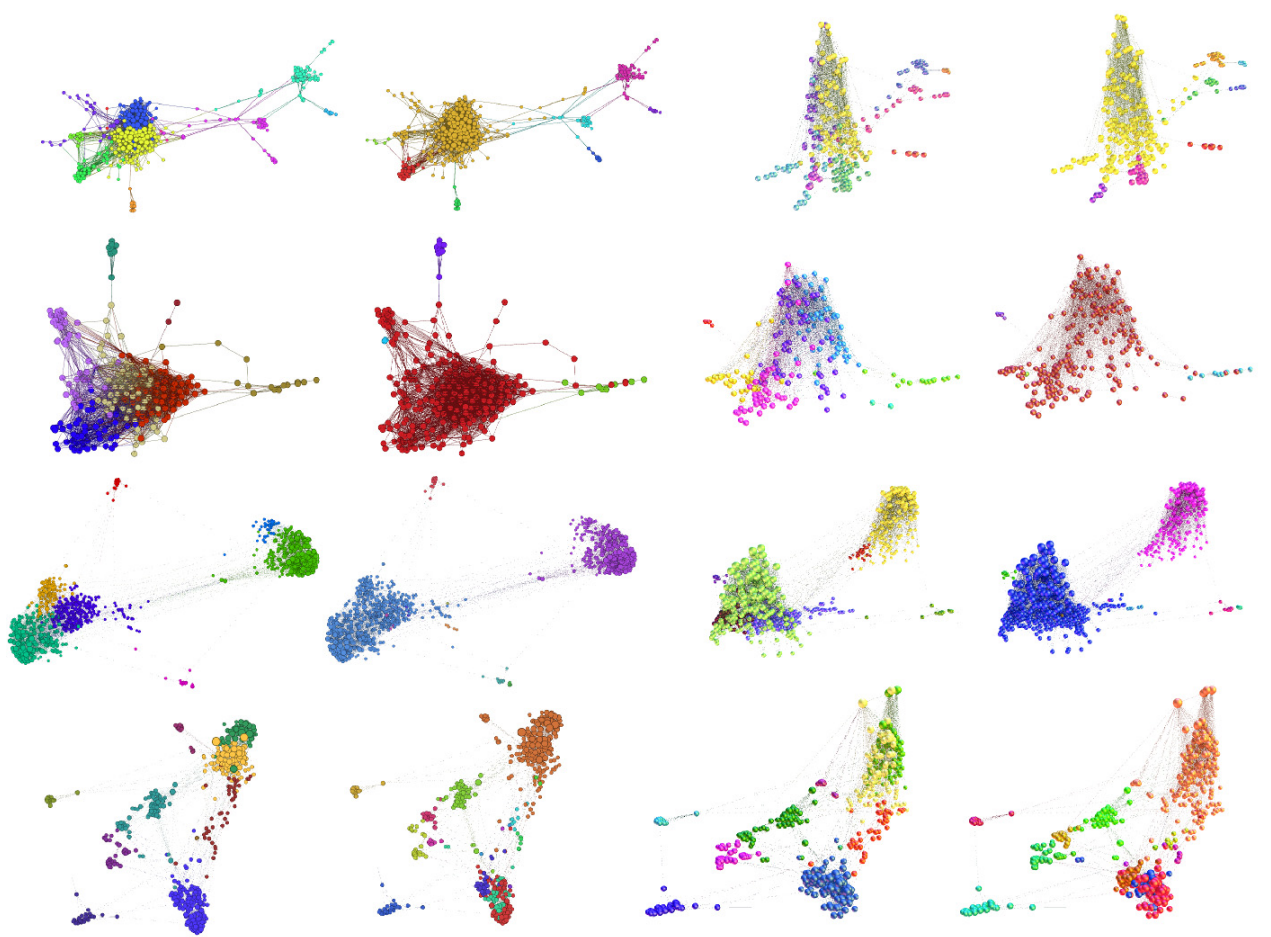

Fig. 7 Figure of facebook network number $0,384,1648$, and 3437. Row - from top to bottom: 0, 384, 1648, 3437. Column - from left to right: Louvain algorithm, our algorithm, Louvain algorithm in 3D, our algorithm in 3D

20th international conference on Parallel and distributed processing, IPDPS'06, pp. 124-124, Washington, DC, USA, (2006) IEEE Computer Society.

[Barabási 03] A.-L. L. Barabási and E. Bonabeau : Scalefree networks. Scientific American, Vol. 288 , No. 5, pp. 60-69, May (2003)

[Blondel 08] V. D. Blondel, J.-L. Guillaume, R. Lambiotte, and E. Lefebvre: Fast unfolding of communities in large networks. Journal of Statistical Mechanics: Theory and Experiment, July (2008).

[Bonacich 07] P. Bonacich. Some unique properties of eigenvector centrality. Social Networks, Vol. 29, No. 4, pp. 555-564, Oct. (2007)

[Borgatti 05] S. Borgatti. Centrality and network flow. Social Networks, Vol. 27, No. 1, pp. 55-71, Jan. (2005)

[Estrada 11] E. Estrada. The Structure of Complex Networks: Theory and Applications: Theory and Applications. OUP Oxford, (2011)

[Estrada 05] E. Estrada and J. A. Rodríguez-Velázquez. Subgraph centrality in complex networks. Phys Rev E Stat Nonlin Soft Matter Phys, Vol. 71, No. 5 Pt 2, May (2005)

[Fortunato 13] S. Fortunato. Benchmark graphs to test community detection algorithms, https://sites.google.com/site/santofortunato/inthepress2.

[Fortunato 10] S. Fortunato. Community detection in graphs. Physics Reports, Vol. 486, pp. 75-174, Jan. (2010)

[Foster 01] K. Foster, S. Muth, J. Potterat, and R. Rothenberg. A Faster Katz Status Score Algorithm. Computational and Mathematical Organization Theory, Vol. 7, No. 4, pp. 275-285, Dec. (2001)

[Freeman 77] L. C. Freeman. A Set of Measures of Centrality Based on Betweenness. Sociometry, Vol. 40, No. 1, pp. 35-41(1977)

[Girvan 02] M. Girvan and M. E. J. Newman. Community structure in social and biological networks. Proceedings of the National Academy of Sciences of the United States of America, Vol. 99, No. 12, pp. 7821-7826, June (2002)

[Jacomy 11] M. Jacomy. Force atlas 2 layout, http://gephi.org/2011/forceatlas2-the-newversion-of-our-homebrew-layout/, (2011)

[Katz 53] L. Katz. A new status index derived from sociometric analysis. Psychometrika, Vol. 18, No. 1, pp. 39-43, Mar. (1953)

[Klein 93] D. J. Klein and M. Randić. Resistance distance. Journal of Mathematical Chemistry, Vol. 12, No. 1, pp. 81-95, Dec. (1993)

[Krebs 04] V. Krebs. Political book networks, http://www.orgnet.com/divided.html, (2004)
[Lancichinetti 09] A. Lancichinetti, S. Fortunato, and J. Kertész. Detecting the overlapping and hierarchical community structure in complex networks. New Journal of Physics, Vol. 11, No. 3, Mar. (2009)

[Lancichinetti 08] A. Lancichinetti, S. Fortunato, and F. Radicchi. Benchmark graphs for testing community detection algorithms. Physical Review E (Statistical, Nonlinear, and Soft Matter Physics), Vol. 78, No. 4 (2008)

[Lusseau 03] D. Lusseau, K. Schneider, O. J. Boisseau, P. Haase, E. Slooten, and S. M. Dawson. The bottlenose dolphin community of Doubtful Sound features a large proportion of long-lasting associations. Behavioral Ecology and Sociobiology, Vol. 54, No. 4, pp. 396405 (2003)

[McAuley 12] J. McAuley and J. Leskovec. Discovering Social Circles in Ego Networks, Oct. (2012).

[Qian 07] R. Qian, W. Zhang, and B. Yang. Community detection in scale-free networks based on hypergraph model. In Proceedings of the 2007 Pacific Asia conference on Intelligence and security informatics, PAISI'07, pp. 226-231, Berlin, Heidelberg, (2007) SpringerVerlag.

[Xu 07] X. Xu, N. Yuruk, Z. Feng, and T. A. J. Schweiger. Scan: a structural clustering algorithm for networks. In Proceedings of the 13th ACM SIGKDD international conference on Knowledge discovery and data mining, KDD '07, pp. 824-833, New York, NY, USA, (2007) ACM.

[Zachary 77] W. W. Zachary. An Information Flow Model for Conflict and Fission in Small Groups. Journal of Anthropological Research, Vol. 33, No. 4, pp. 452-473 (1977)

\section{〔担当委員 : TORIUMI, Fujio〕}

Received April 2, 2013. 


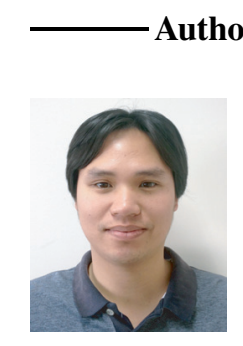

\section{Author's Profile}

\section{Sorn Jarukasemratana}

Currently a Ph.D. student at Tokyo Institute of Technology under the supervision of Dr. Tsuyoshi Murata. He graduated his master degree from University of Tampere, Finland with M.Sc. in User Interface Software Development. His bachelor degree is obtained from faculty of engineering, Chulalongkorn University, Thailand with computer engineering major.

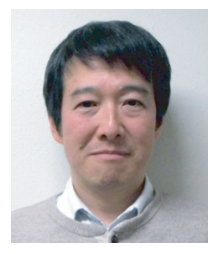

\section{Tsuyoshi Murata, Dr. (Member)}

$\mathrm{He}$ is an associate professor in the Department of Computer Science, Graduate School of Information Science and Engineering, Tokyo Institute of Technology. He obtained his doctor's degree in Computer Science at Tokyo Institute of Technology in 1997, on the topic of Machine Discovery of Geometrical Theorems. At Tokyo Institute of Technology, he conducts research on Web mining and social network analysis.

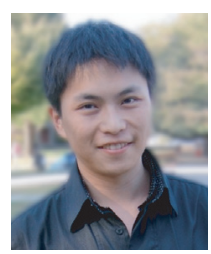

\section{Xin Liu, Dr.}

Xin Liu received a M.Sc. degree in computer science from Wuhan University in 2007 and a Ph.D. degree in computer science from Tokyo Institute of Technology in 2011. He is now a research fellow at Tokyo Institute of Technology (JST CREST) and an associate professor at Wuhan University of Technology. His research interests include web mining and social network analysis. He is a member of ACM. 